\title{
Abstract: Automatic Estimation of Cochlear Duct Length and Volume Size
}

\author{
Ibraheem Al-Dhamari ${ }^{1}$, Sabine Bauer ${ }^{1}$, Dietrich Paulus ${ }^{1}$, Rania Hilal ${ }^{2}$, \\ Friedrich Lissek ${ }^{3}$, Roland Jacob ${ }^{3}$ \\ ${ }^{1}$ Koblenz and Landau University, Koblenz \\ ${ }^{2}$ Germany Ain Shams University, Cairo, Egypt \\ ${ }^{3}$ Military Hospital, Koblenz, Germany \\ idhamari@uni-koblenz.de
}

The exact cochlear length and size are required is an important factor of selecting the suitable cochlear implant. We present a fast cochlear length and volume size estimation method from clinical multi-modal medical images. The method utilizes atlas-model-based segmentation to estimate a transformation from a model to an input volume. The result is used to transform a well-defined segmentation and a points-set of a scala tympani to the input image that segments and estimates the scala tympani length in a few seconds using standard hardware e.g. a laptop. The method is based on automatic cochlea image registration (ACIR) [1]. The error is estimated using the known length of the cochlear implants. A dataset of $713 \mathrm{D}$ images of 21 patients from various age and gender groups is used. The estimated average scala tympani length was $29.54 \mathrm{~mm}$, with $0.27 \mathrm{~mm}$ standard deviation. The average scala tympani volume size was $41.56 \mathrm{~mm}^{3}$, with $0.19 \mathrm{~mm}^{3}$ standard deviation (Fig. 1). The method is available as an open source 3D Slicer plug-in. The source code and the data can can be downloaded from a public server as described in [2].
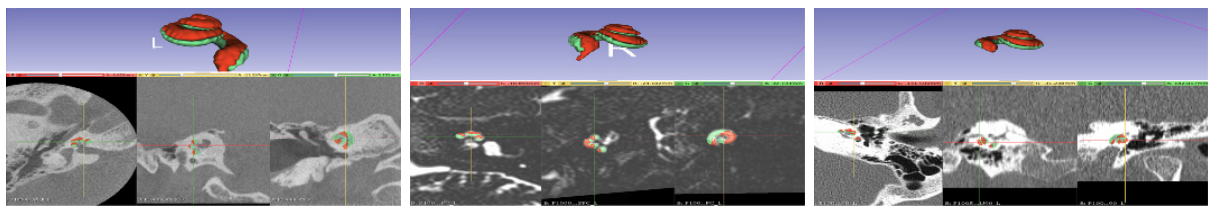

Fig. 1. Samples of segmentation results, left: CBCT, middle: MR and right: CT.

\section{References}

1. Al-Dhamari I, Bauer S, Paulus D, et al. ACIR: automatic cochlea image registration. Proc SPIE. 2017;10133(10):1-5.

2. Al-Dhamari I, Bauer S, Paulus D, et al. Automatic cochlear length and volume size estimation. OR 20 Context-Aware Oper Theaters, Comput Assist Rob Endosc. 2018 ; p. 54-61. 